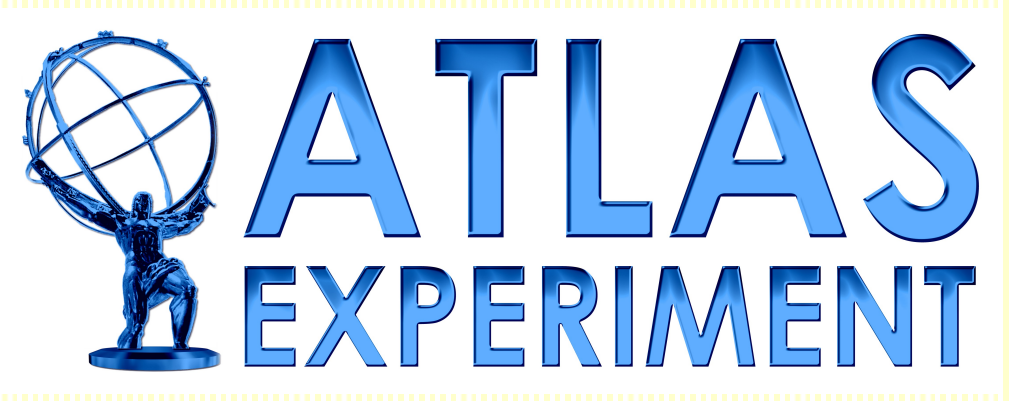

\title{
Search for Dark Matter Candidate with the ATLAS detector
}

\section{Marie-Hélène Genest (LMU Munich) \\ On behalf of the ATLAS Collaboration RICAP 2011}
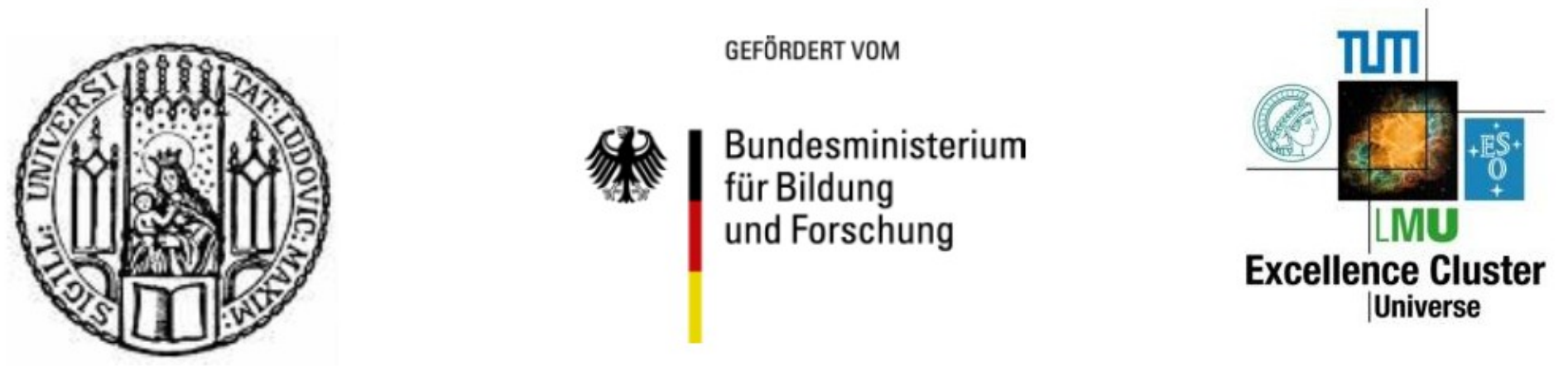


\section{Spin $1 / 2$}
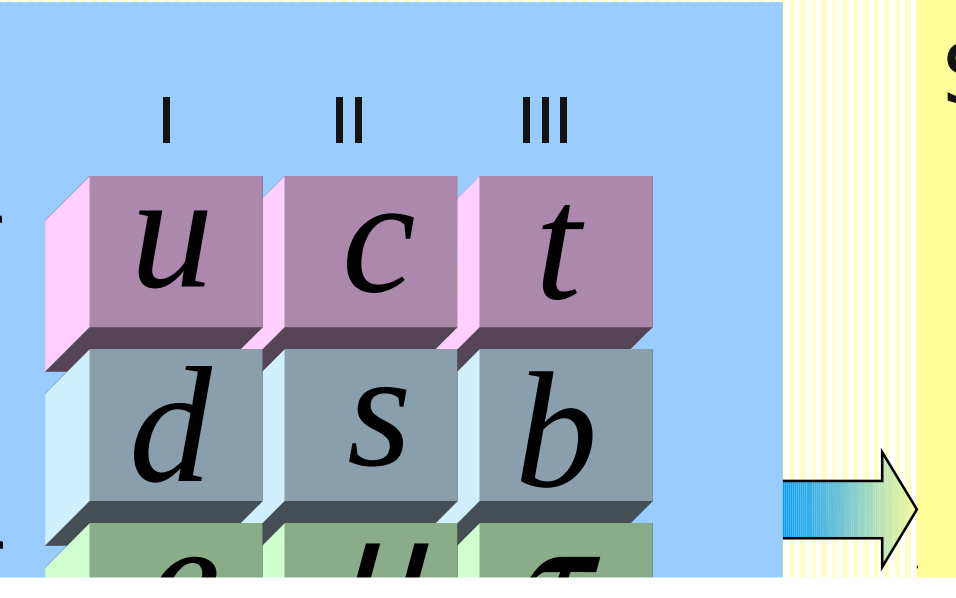

Spin 0

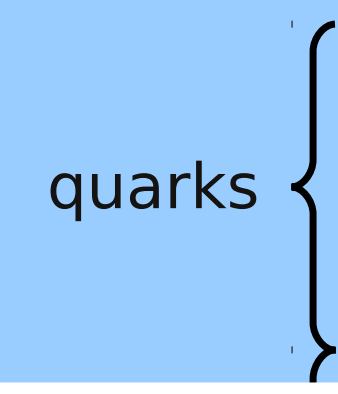

If R-parity $(-1)^{L+3 B+2 S}$ is conserved:

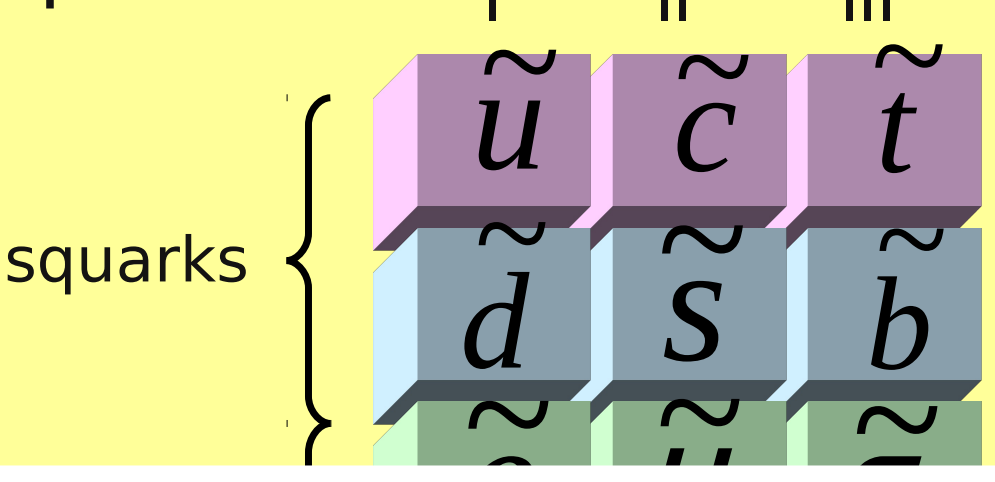

- SM particles have $\mathrm{R}=+1$

- SUSY particles have $\mathrm{R}=-1$

The lightest sparticle (LSP) is stable: Dark matter candidate!

(u

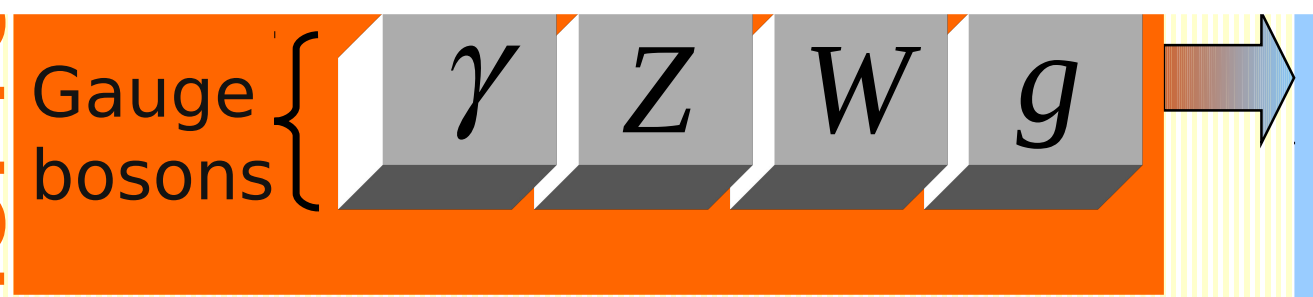

Spin 0

$\underset{\text { bosons }}{\text { Higgs }}\left\{h, H H^{ \pm} A\right.$ gluino $\{\tilde{g}$ Neutralinos $\left\{\tilde{\chi}_{1}^{0} \tilde{\chi}_{2}^{0} \tilde{\chi}_{3}^{0} \tilde{\chi}_{4}^{0}\right.$ Charginos $\begin{cases}\tilde{\chi}_{1}^{ \pm} & \tilde{\chi}_{2}^{ \pm} \\ \end{cases}$ 


\section{Typical signature}

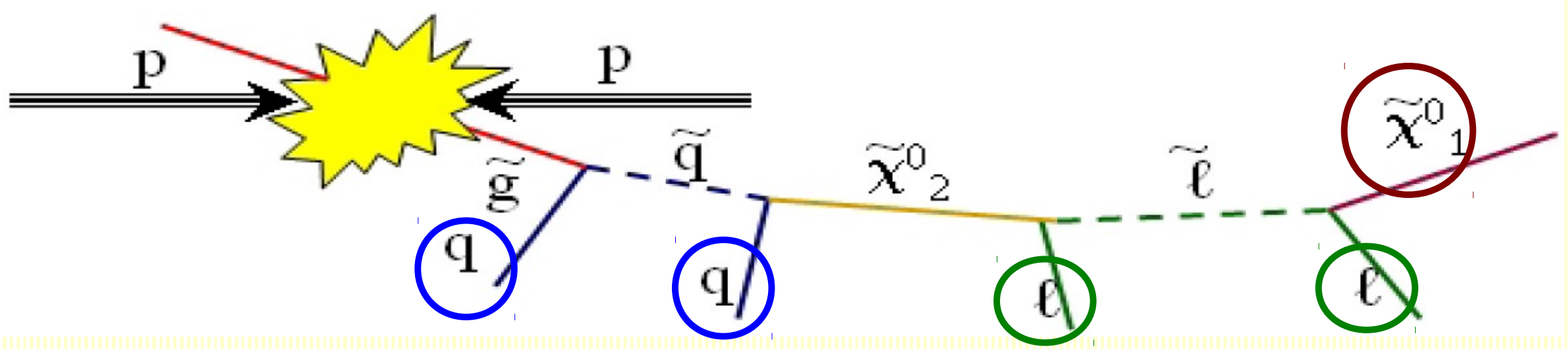

- Pair of gluinos/squarks produced by strong interactions

- Their decays give high- $p_{T}$ jets and charginos/neutralinos

- Charginos/neutralinos decays can give leptons and the decay chain stops when the LSP is produced (R-parity conserving scenarios)

- The pair of stable LSP produced escapes the detector undetected leading to high transverse missing energy

\section{multi-Jets $+\mathrm{n}$ leptons $+\mathrm{E}_{\mathrm{T}}^{\text {miss }}$}

Standard Model backgrounds (tt, W+jets, Z+jets, QCD jets and dibosons) 


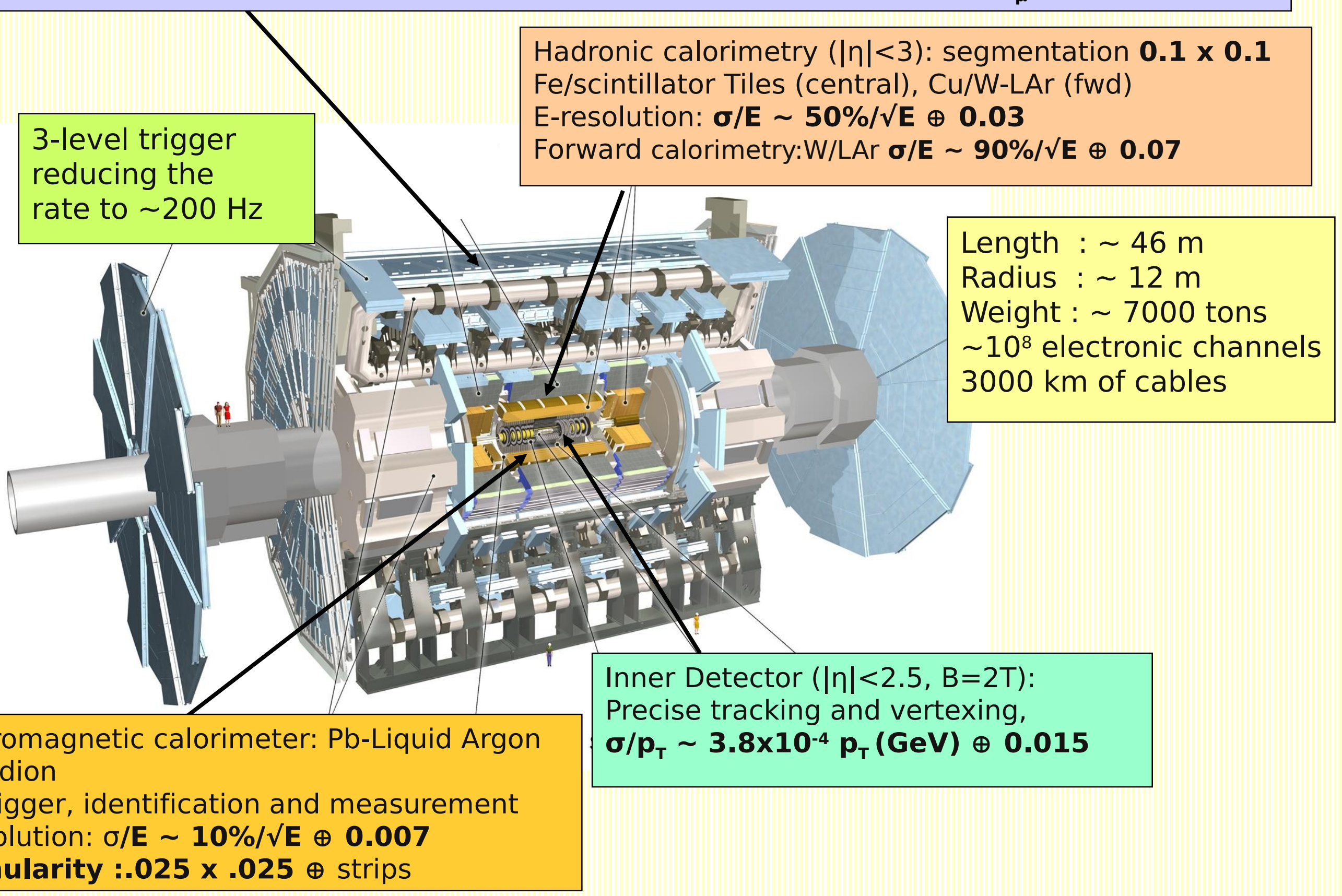

Electromagnetic calorimeter: Pb-Liquid Argon Accordion

e/ $/$ trigger, identification and measurement E-resolution: $\sigma / \mathrm{E} \sim$ 10\%/VE $\oplus 0.007$

granularity :.025 x.025 $\oplus$ strips 


\section{Data accumulated in 2010}

- Excellent LHC performance
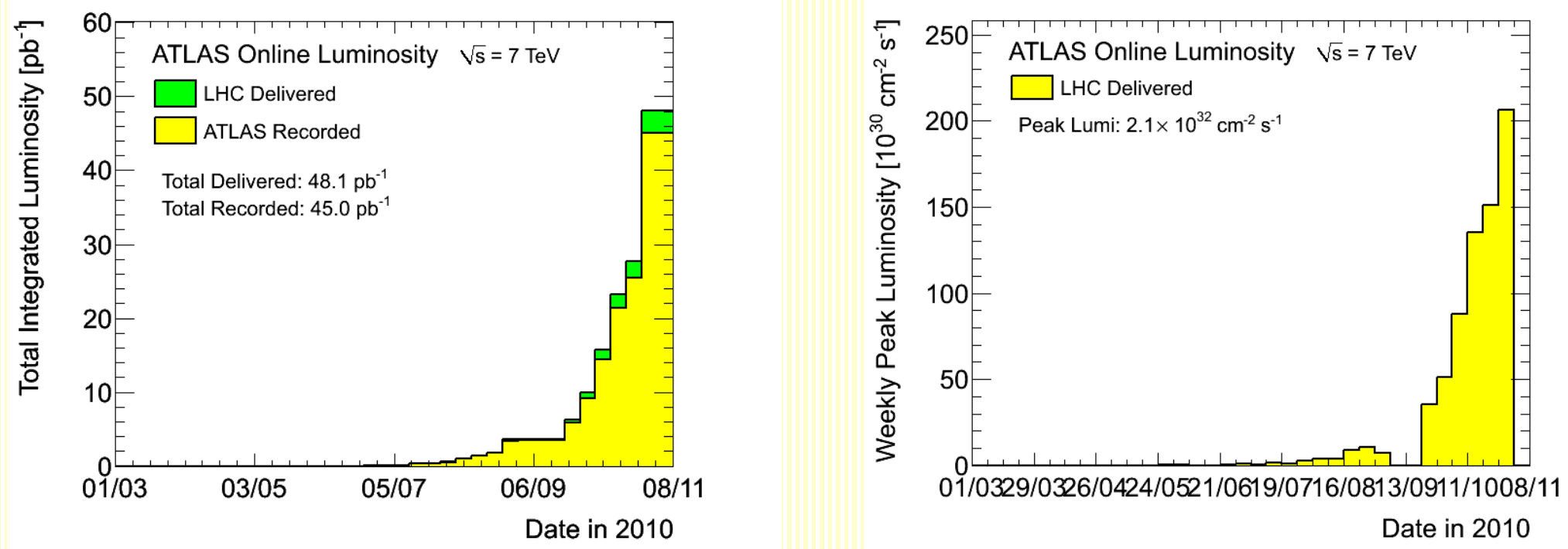

- Very good detector efficiency:

- Inner tracking detectors: $99.1 \%$ to $100 \%$

- Calorimeters: $90.7 \%$ to $100 \%$

- Muon detectors: $96.2 \%$ to $99.8 \%$ 


\section{ATLAS public results of R-parity conserving SUSY searches}

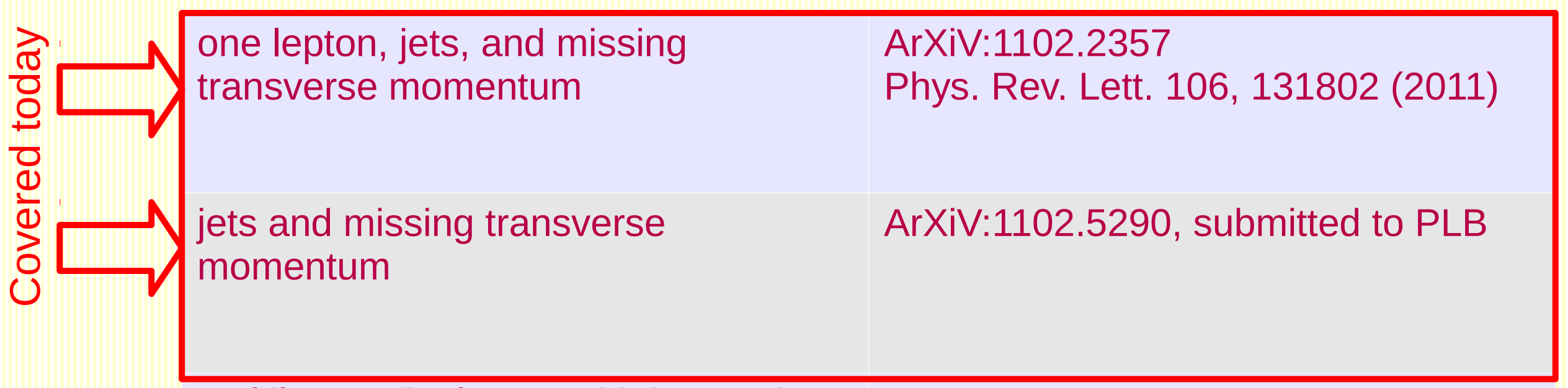

Multilepton final state with jets and ATLAS-CONF-2011-039

missing transverse momentum

b-tagged jets and missing transverse ArXiV:1103.4344, submitted to PLB momentum

Dilepton final states with missing transverse momentum

ArXiV:1103.6214, submitted EPJLC

Same-flavour:

ArXiV:1103.6208, accepted EPJLC 


\section{Some useful variables}

- $\Delta \phi\left(j e t s, \mathrm{E}_{\mathrm{T}}^{\text {miss }}\right)$

- Cutting on $\Delta \phi$ eliminates events in which $\mathrm{E}_{t}^{\text {miss }}$ is closely related to one of the leading jets (QCD)

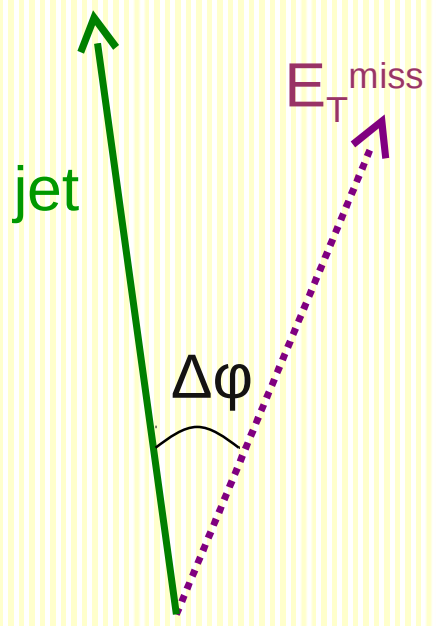

- Effective mass $m_{\text {eff }}$ (scalar sum of sel. jets \& leptons $p_{T}$ and $E_{T}^{\text {miss }}$ )

- peaks at a value which is correlated with the mass of the pair of SUSY particles produced in the $p p$ interaction

- The transverse mass $m_{\mathrm{T}} m_{\mathrm{T}}^{2} \equiv 2\left|\mathbf{p}_{\mathrm{T}}^{\mathrm{P}}\right|\left|E_{\mathrm{T}}^{\mathrm{m} \mid \mathrm{ss}}\right|-2 \mathbf{p}_{\mathrm{T}}^{\mathrm{\ell}} \cdot E_{\mathrm{T}}^{\overrightarrow{\mathrm{m}} \mathrm{uss}}$

- useful to remove $B G$ in which a $W$ decays leptonically 


\section{The 0-lepton channel}

Select events with jets, missing transverse momentum and no lepton (veto e/ $\mu$ )

arXiV:1102.5290

submitted to PLB 


\section{Defining the signal regions}

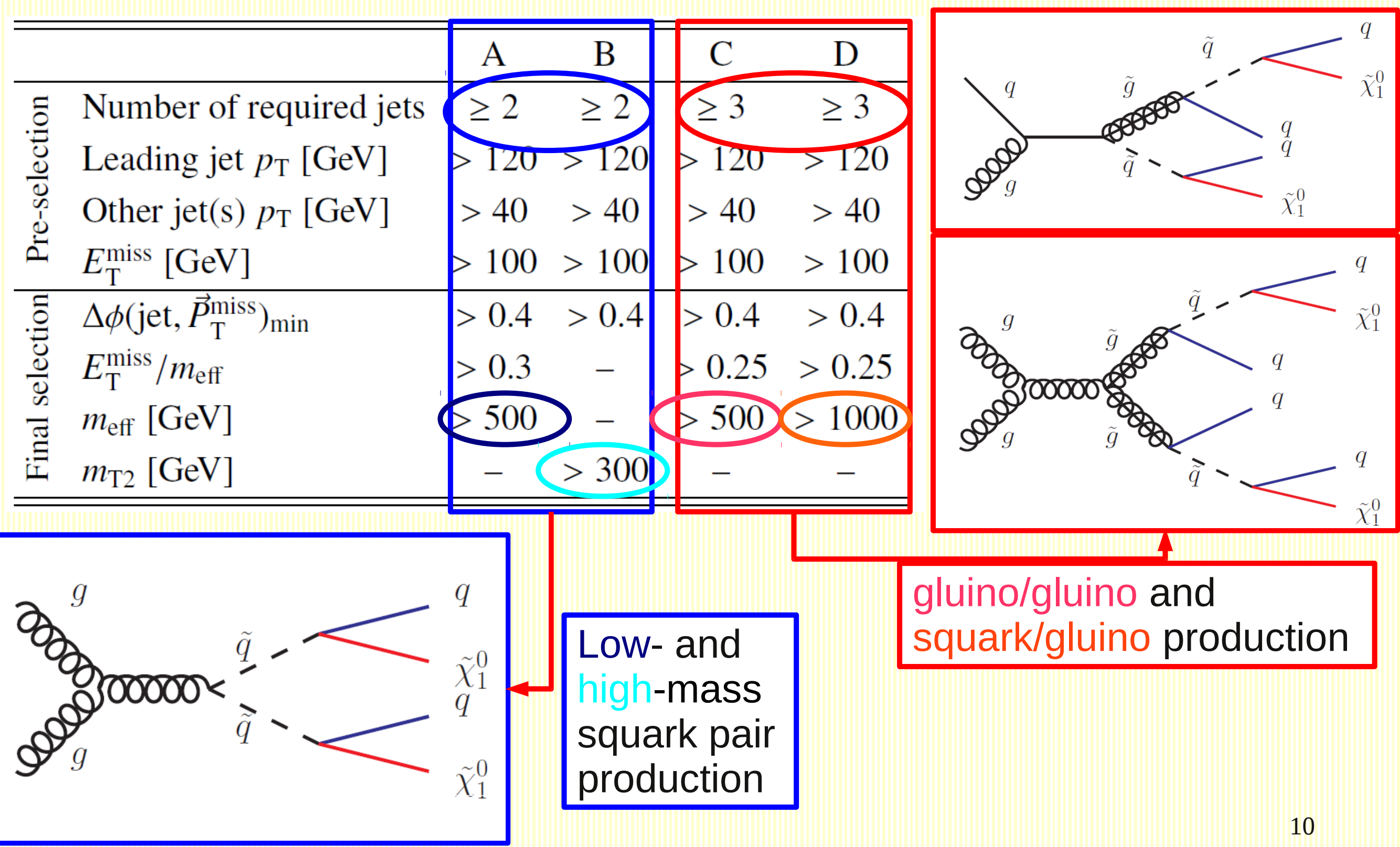




\section{Defining the signal regions}

\begin{tabular}{|c|c|c|c|c|c|}
\hline & & $\bar{A}$ & $\mathrm{~B}$ & $\mathrm{C}$ & $\bar{D}$ \\
\hline \multirow{4}{*}{ 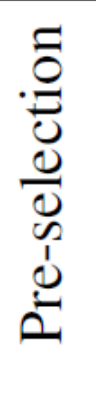 } & Number of required jets & $\geq 2$ & $\geq 2$ & $\geq 3$ & $\geq 3$ \\
\hline & Leading jet $p_{\mathrm{T}}[\mathrm{GeV}]$ & $>120$ & $>120$ & $>120$ & $>120$ \\
\hline & Other jet(s) $p_{\mathrm{T}}[\mathrm{GeV}]$ & $>40$ & $>40$ & $>40$ & $>40$ \\
\hline & $E_{\mathrm{T}}^{\text {miss }}[\mathrm{GeV}]$ & $>100$ & $>100$ & $>100$ & $>100$ \\
\hline \multirow{4}{*}{ 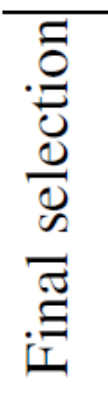 } & $\Delta \phi\left(\text { jet, }, \bar{P}_{\mathrm{T}}^{\mathrm{miss}}\right)_{\min }$ & $>0.4$ & $>0.4$ & $>0.4$ & $>0.4$ \\
\hline & $E_{\mathrm{T}}^{\mathrm{miss}} / m_{\mathrm{eff}}$ & $>0.3$ & - & $>0.25$ & $>0.25$ \\
\hline & $m_{\mathrm{eff}}[\mathrm{GeV}]$ & $>500$ & $\overline{-}$ & $>500$ & $>1000$ \\
\hline & $m_{\mathrm{T} 2}[\mathrm{GeV}]$ & - & $>300$ & - & - \\
\hline
\end{tabular}

Trigger requirements

Reject the QCD BG

Optimize for SUSY

Stranverse mass $\mathrm{m}_{\mathrm{T} 2}$ :

- The sum of the transverse missing momentum of the two neutralinos is know: $\vec{q}_{T}^{(1)}+\vec{q}_{T}^{(2)}=E_{T}^{m i s s}$

- Using this constraint, calculate the stransverse mass as:

$m_{T 2}\left(\vec{p}_{T}^{(1)}, \vec{p}_{T}^{(2)}, \vec{p}_{T}\right) \equiv \min _{\dot{q}_{T}^{(1)}+q_{T}^{(2)}=E_{T}^{m m^{m s}}}\left\{\max \left(m_{T}\left(\vec{p}_{T}^{(1)}, \vec{q}_{T}^{(1)}\right), m_{T}\left(\vec{p}_{T}^{(2)}, \vec{q}_{T}^{(2)}\right)\right)\right\}$ 


\section{Main backgrounds}

$W+$ jets: $\tau \nu$ decay or missed e/ $\mu$ Z+jets: $v v$ decay

Alpgen with NNLO normalization

Top pair production: $\tau$ decay of a $W\}$ MC@NLO

\section{Cross-check on data:}

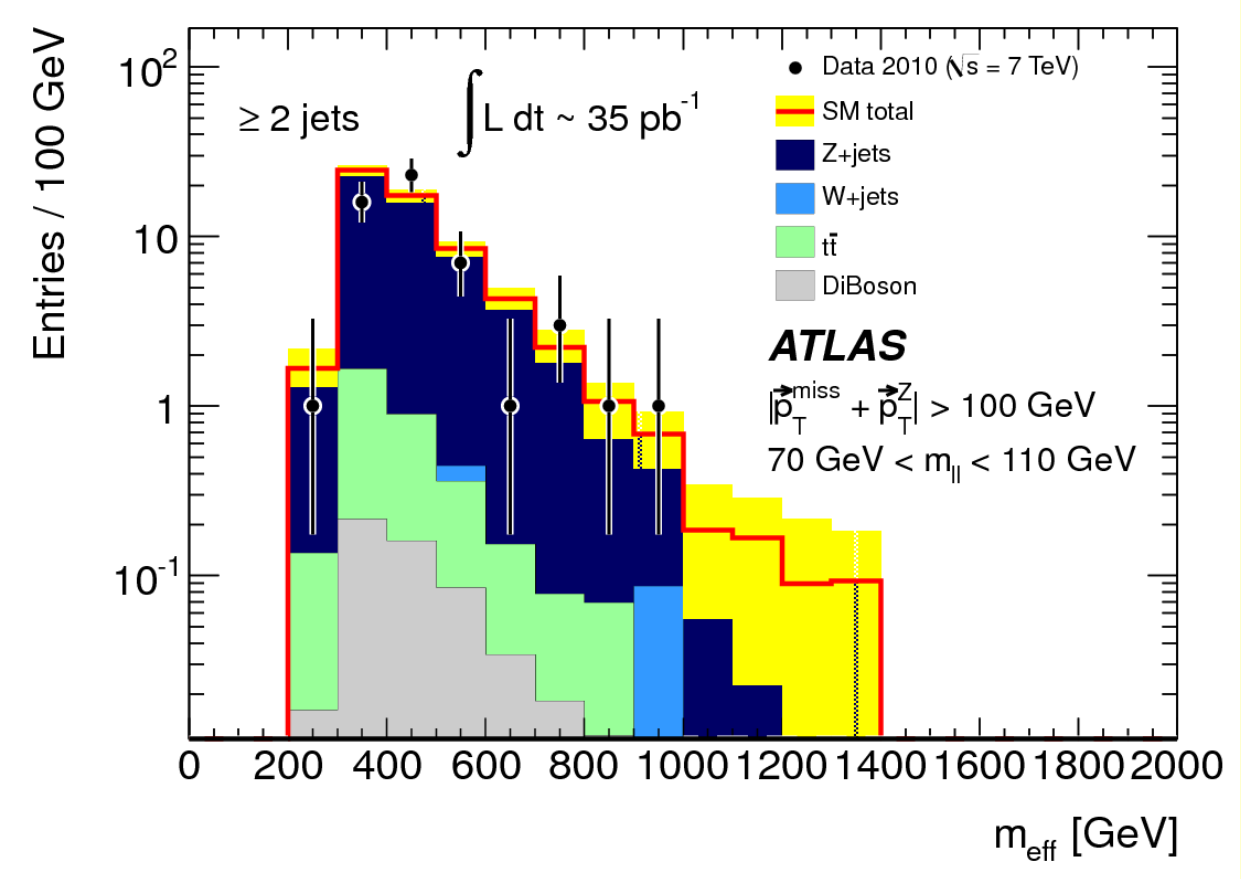

$Z(I I)+$ jets control sample with leptons removed to recalculate the missing transverse momentum

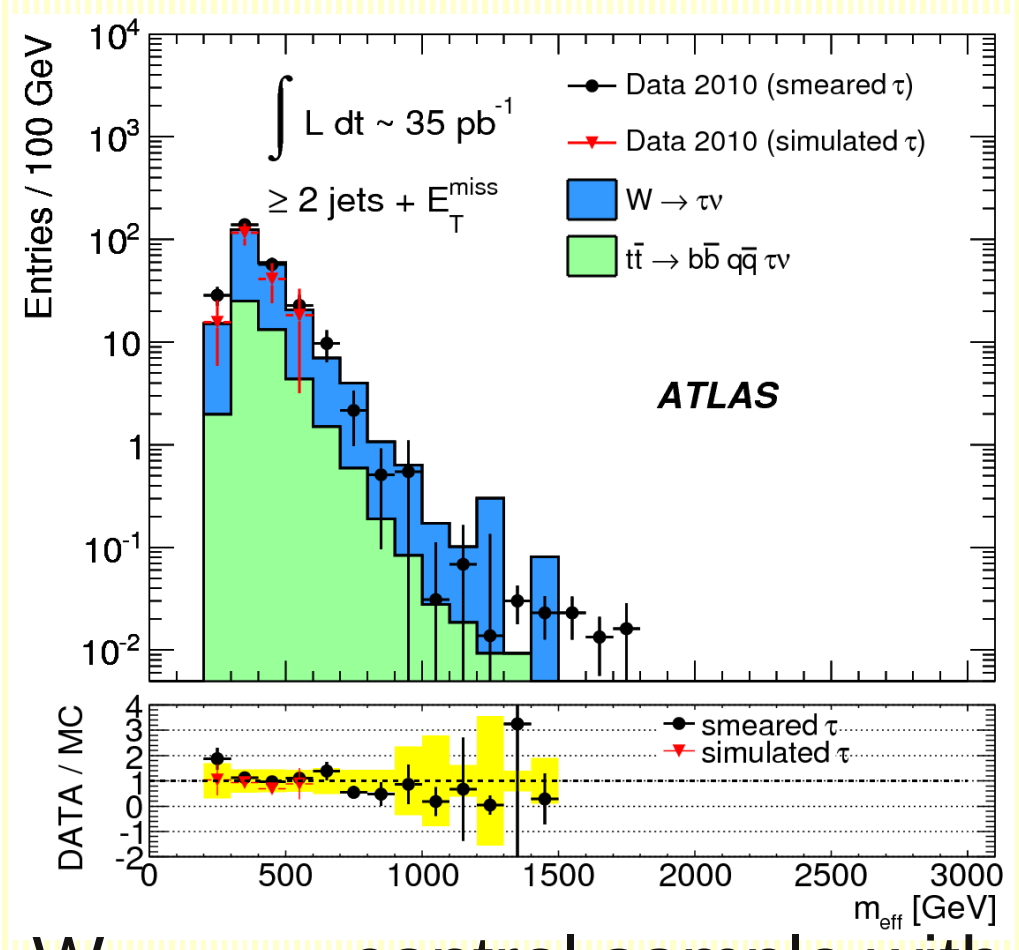

$\mathrm{W} \rightarrow \mu \nu$ control sample with the muon replaced by a MC $\tau$ decay 


\section{Results}
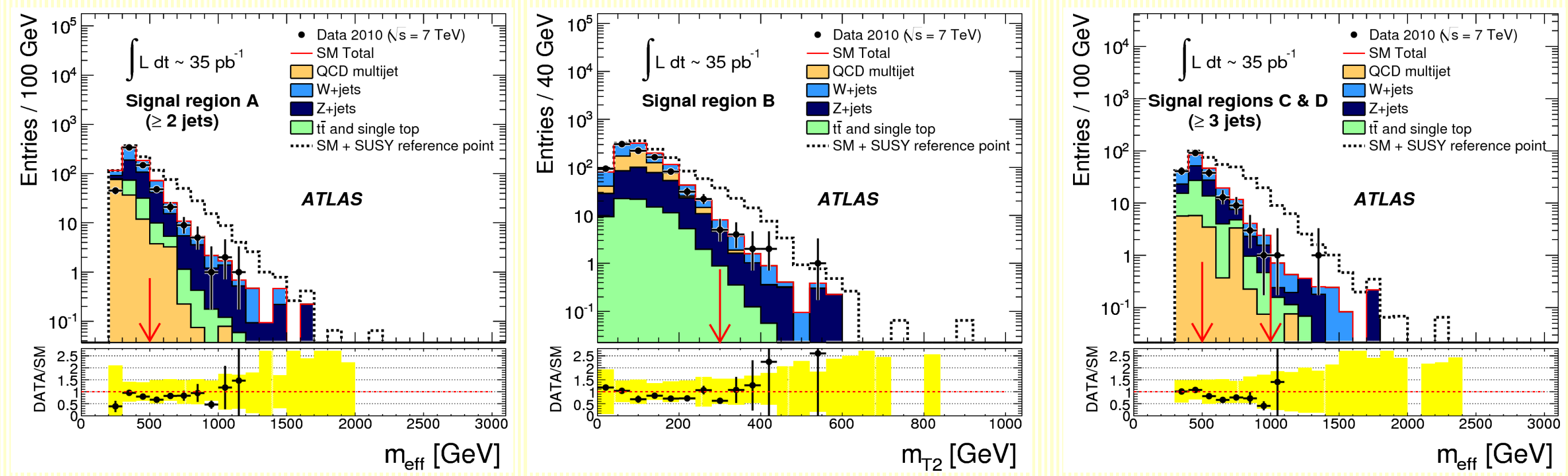

95\% CL limits on cross section - acceptance - efficiency:

$1.3 \mathrm{pb}(\mathrm{A}), 0.35 \mathrm{pb}(\mathrm{B}), 1.1 \mathrm{pb}(\mathrm{C})$ and $0.11 \mathrm{pb}(\mathrm{D})$ 


\section{Exclusion plot}

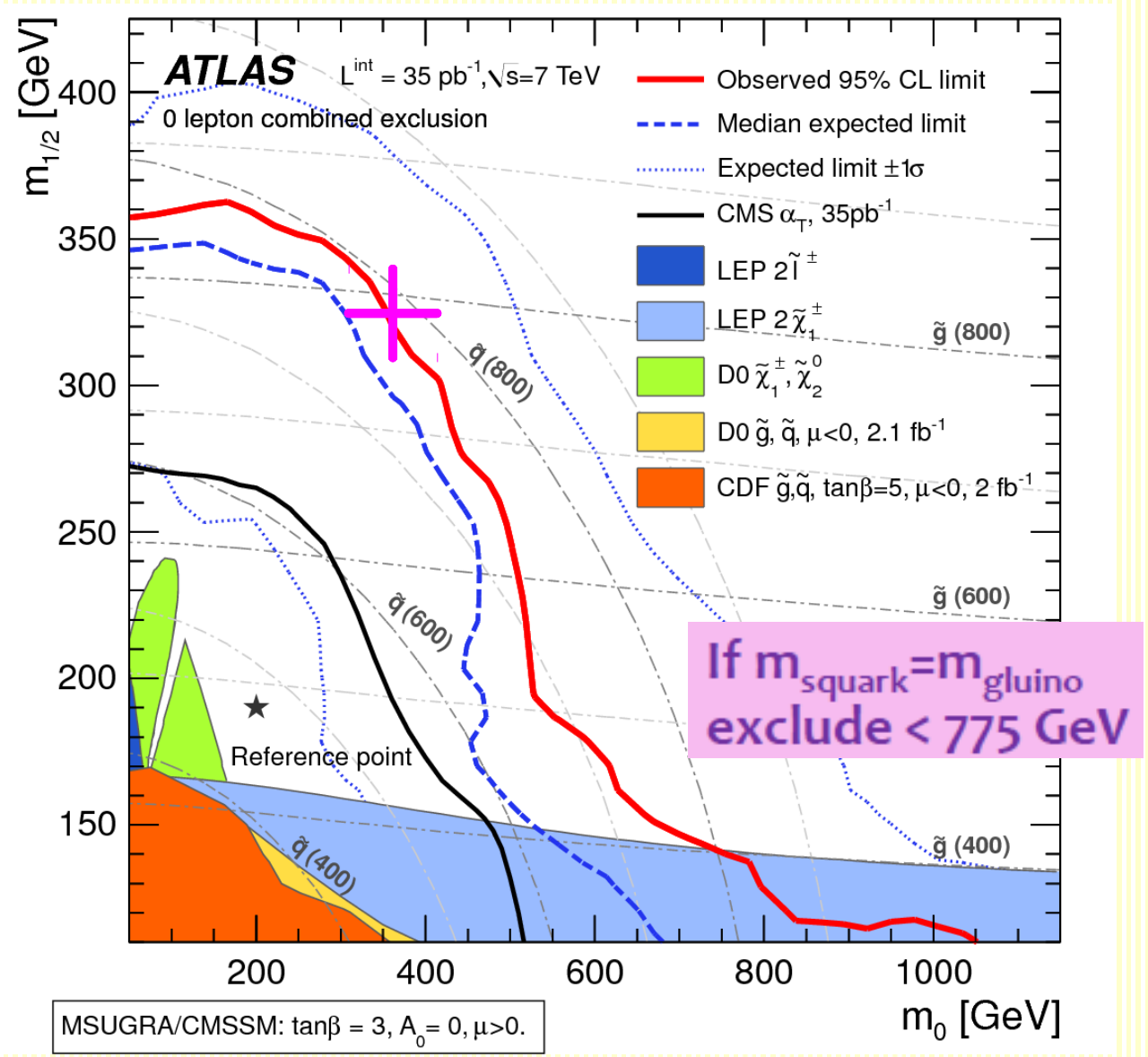

The limit does not depend strongly on $\tan \beta$, A0 or $\operatorname{sign}(\mu)$ 


\title{
The 1-lepton channel
}

Select events with jets, missing transverse momentum and exactly one lepton $(e / \mu)$

\author{
ArXiV:1102.2357 \\ Phys. Rev. Lett. 106, 131802 (2011)
}




\section{Defining the signal region}

The isolated one-lepton requirement suppresses

QCD multijet and allows a lepton-based trigger

- Exactly one lepton $(e / \mu)$ with $p T>20 \mathrm{GeV}$

- At least 3 jets with pT>60,30,30 GeV

- $\Delta \phi\left(\right.$ jets, $\left.\mathrm{E}_{\mathrm{T}}^{\text {miss }}\right)>0.2$

$-E_{T}^{\text {miss }} / m_{\text {eff }}>0.25$

- $m_{T}>100 \mathrm{GeV}$

$-m_{\text {eff }}>500 \mathrm{GeV}$

gluino/squark cascade decay with intermediate steps

Reduce the QCD BG further

Suppresses $\mathrm{W}+$ jets and $\mathrm{tt}$

Optimize for SUSY 


\section{Main backgrounds: $\mathrm{W}+$ jets and $\mathrm{tt}$}

W control region: no b-jet
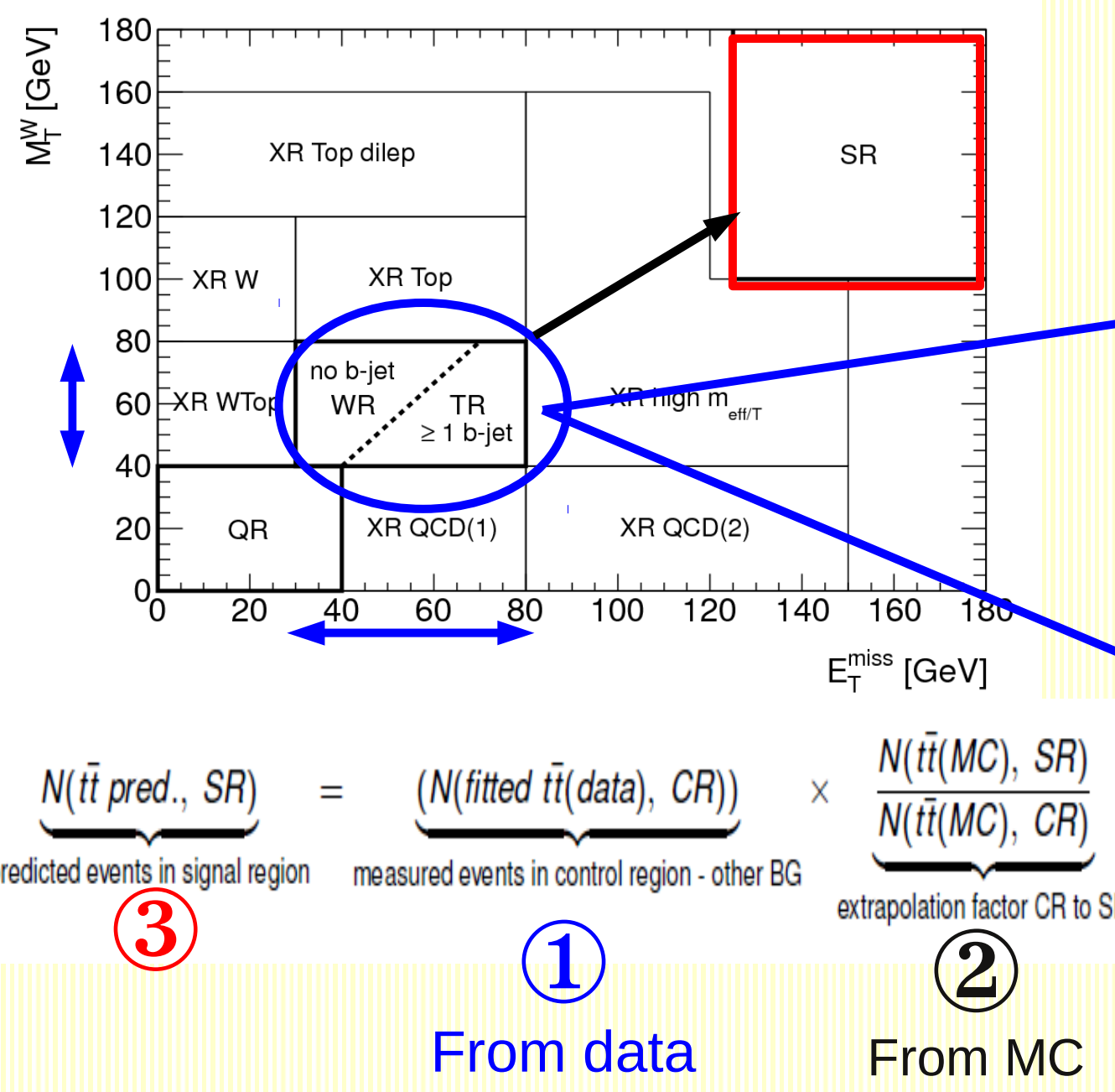

Method cross-checked with additional control regions

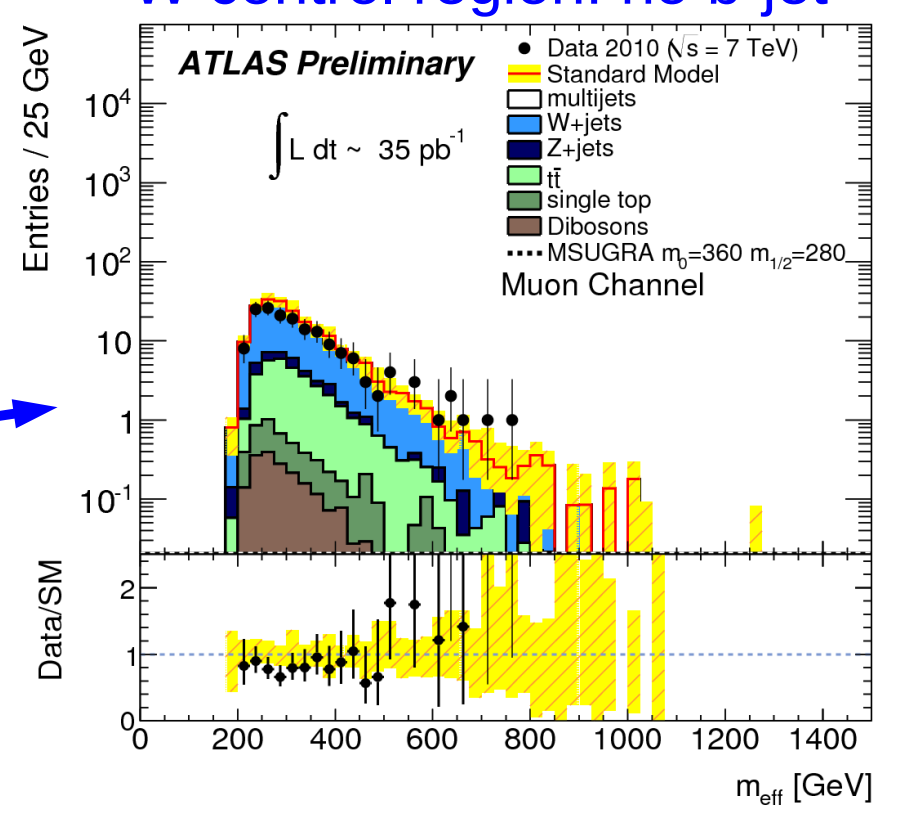

Top control region: $\geq 1$ b-jet

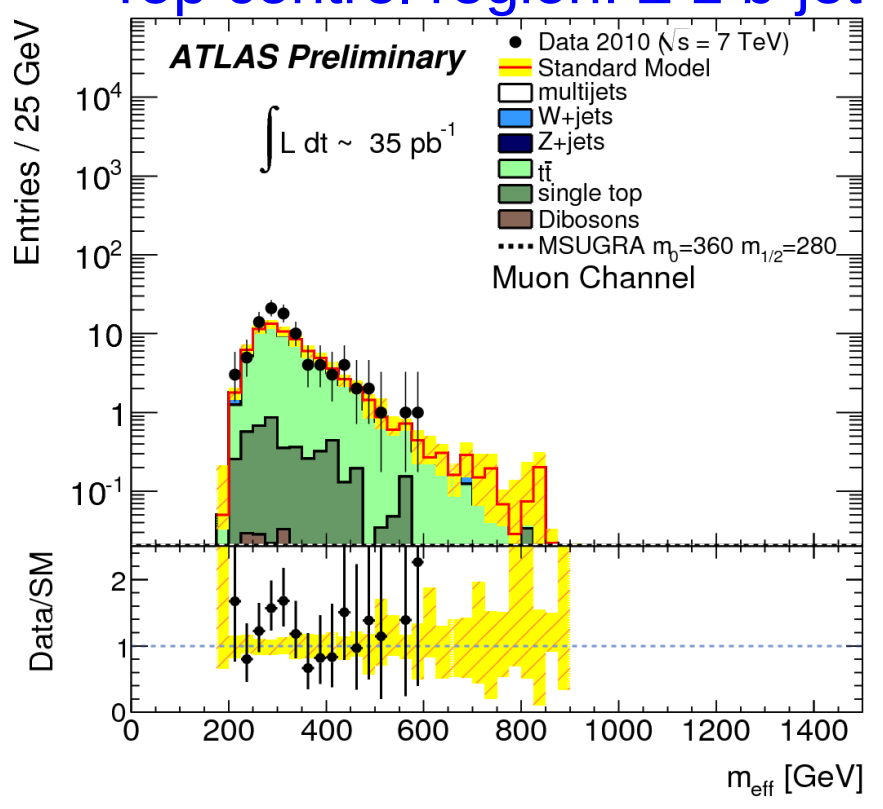




\section{Results}

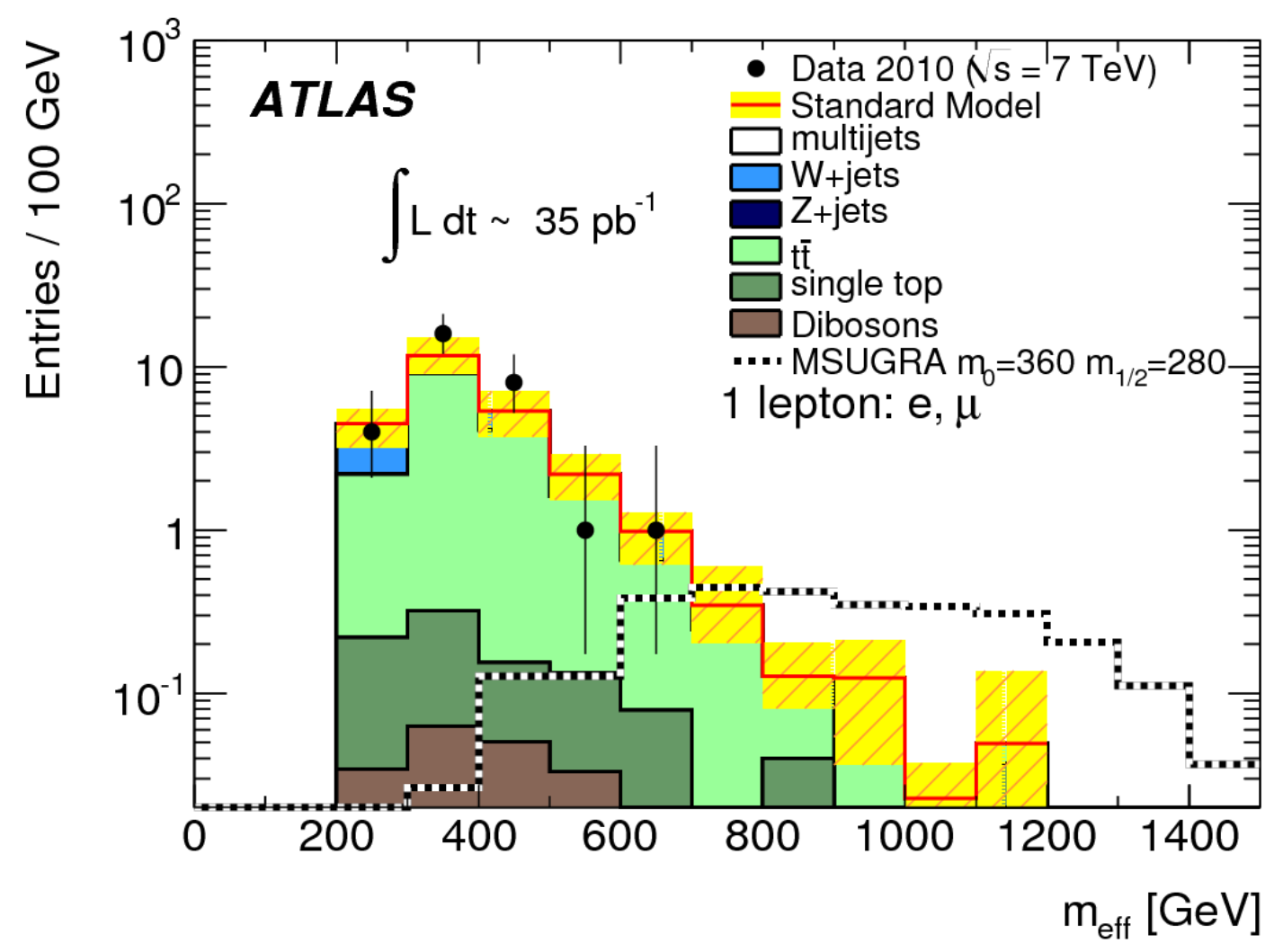

95\% CL limits on cross section - acceptance - efficiency: $0.065 \mathrm{pb}$ (electron), $0.073 \mathrm{pb}$ (muon) 


\section{Exclusion plot}

MSUGRA/CMSSM: $\tan \beta=3, A=0, \mu>0$

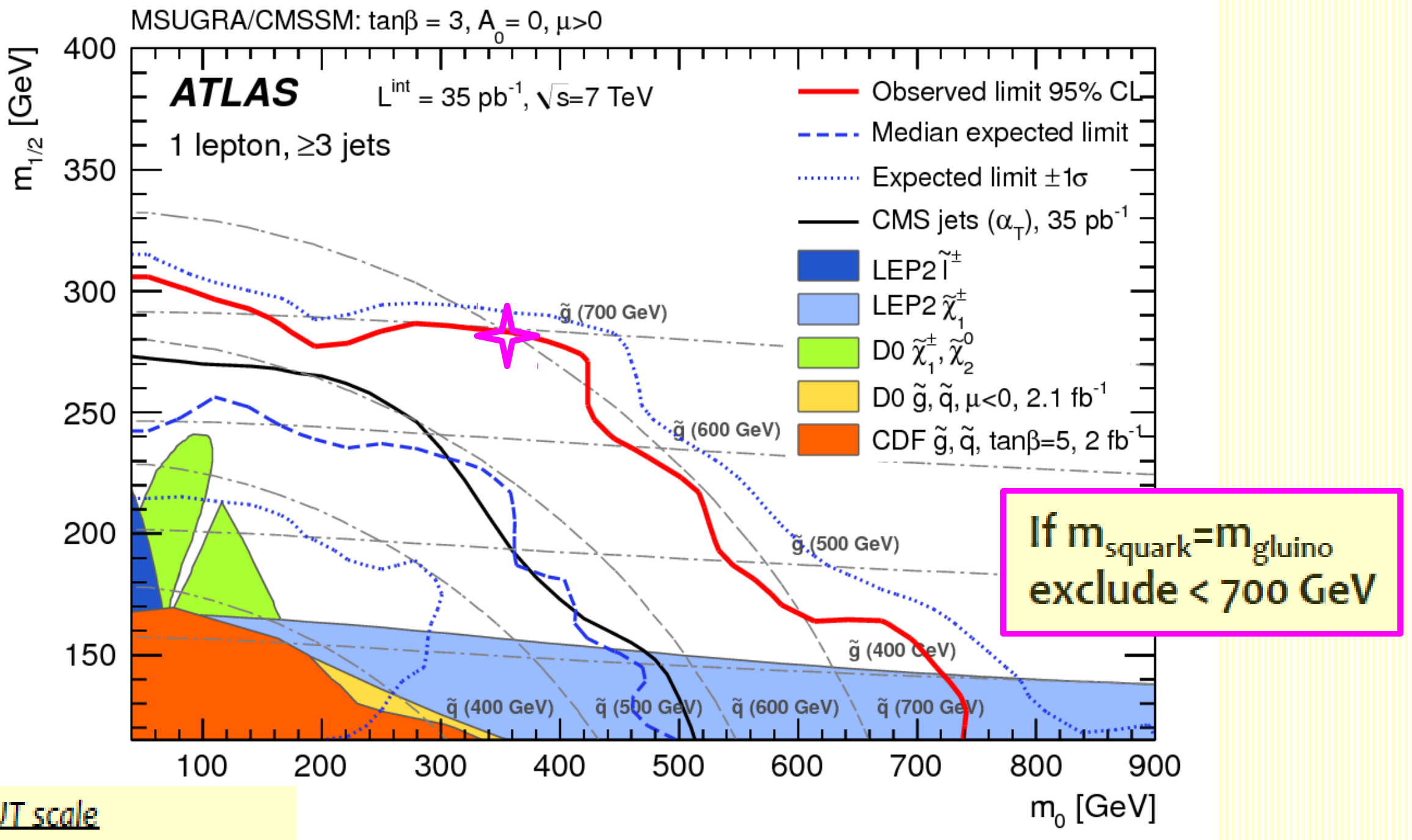

parameters at GUT scale

1. Unified gaugino(scalar) mass $\mathrm{m}_{4 / 2}\left(\mathrm{~m}_{0}\right)$

3. Ratio of $\mathrm{H}_{4}, \mathrm{H}_{2}$ vevs tan $\beta$

4. Trilinear coupling $A_{0}$ 


\section{0- and 1-lepton combination}

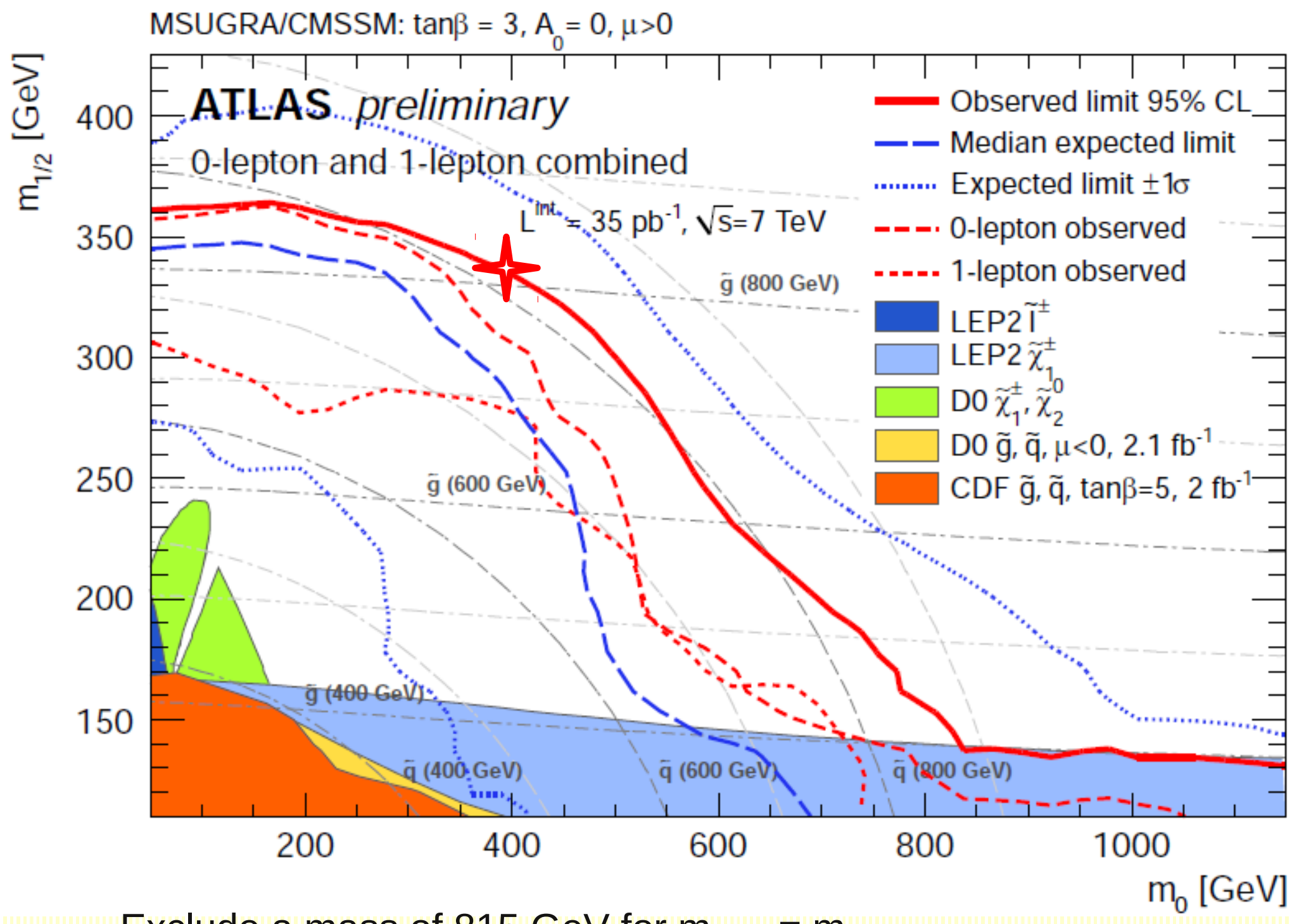

Exclude a mass of $815 \mathrm{GeV}$ for $\mathrm{m}_{\text {gluino }}=\mathrm{m}_{\text {squark }}$ 


\section{How to use the data}

- For each signal region and analysis channel, the efficiency $x$ acceptance is provided

- The LHA SUSY files are provided

$\rightarrow$ validate your setup

$\rightarrow$ interpret the data in your model

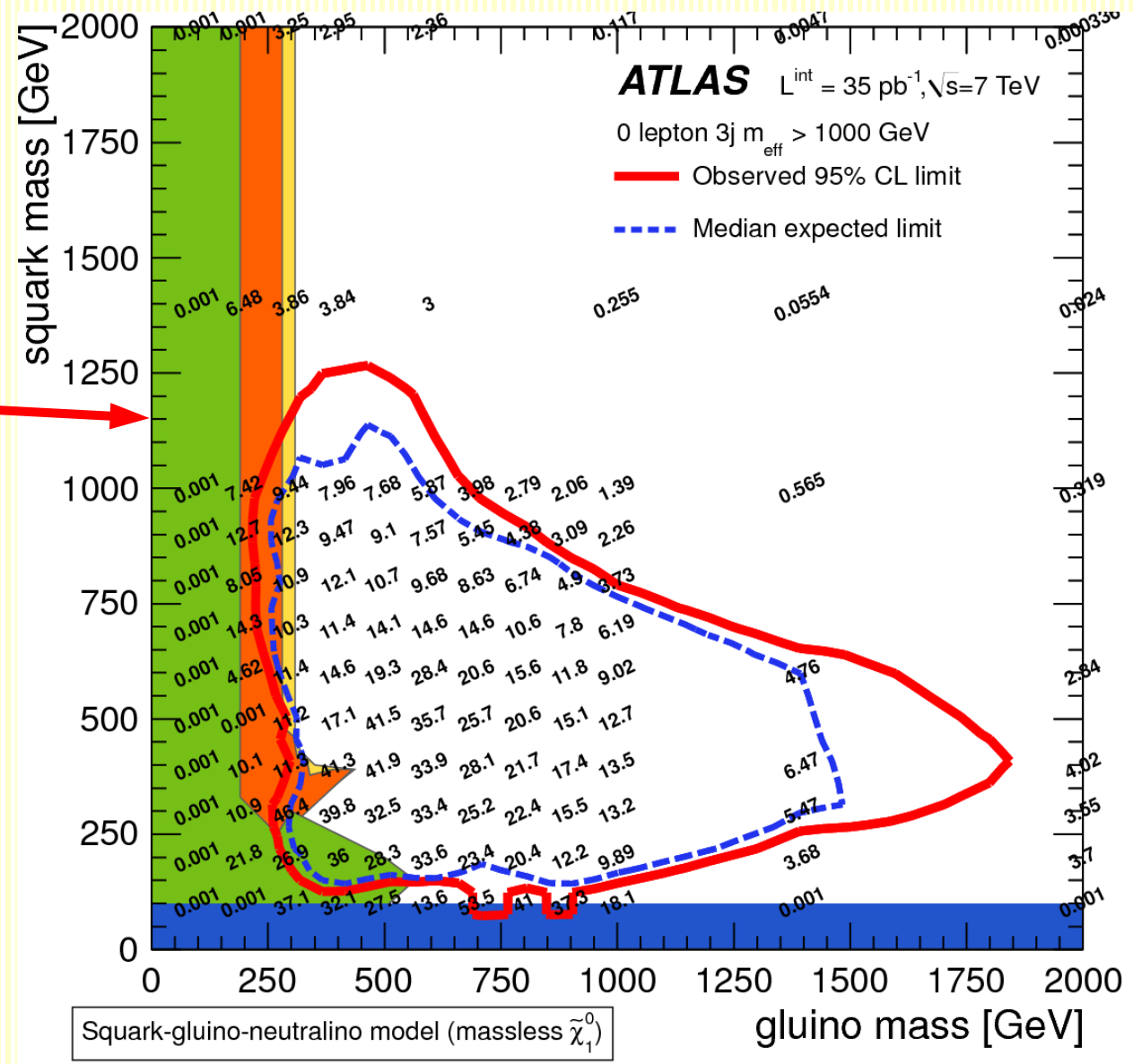




\section{Summary and outlook}

- The search is on!

- ATLAS has already produced many results (in some scenarios the most stringent limits to date) in various channels and more are in the pipeline

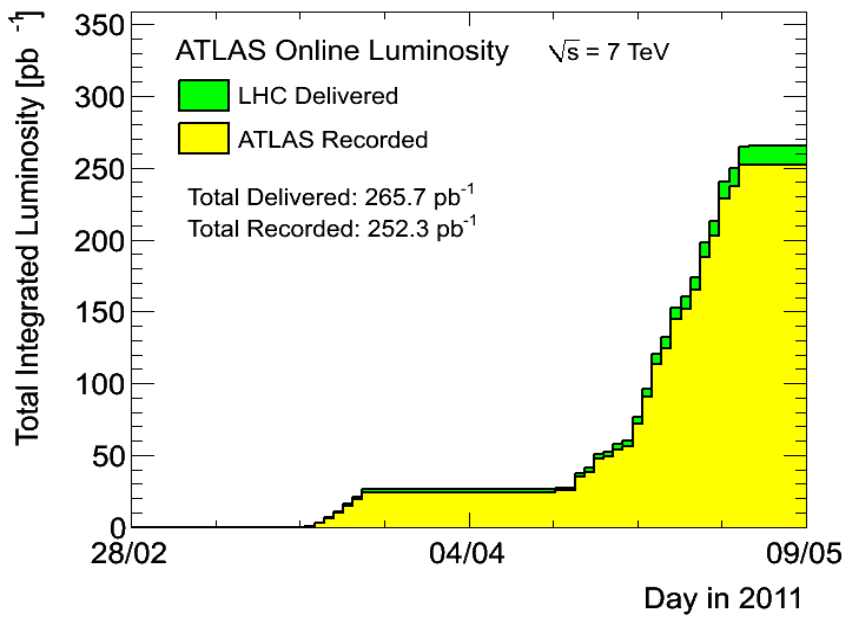

- Already $250 \mathrm{pb}^{-1}$ accumulated in 2011 with a new record luminosity of $8.3 \times 10^{32} \mathrm{~cm}^{-2} \mathrm{~s}^{-1}$ !

- In 2011: more than $1 \mathrm{fb}^{-1}$ of data with luminosities on the order of $10^{33} \mathrm{~cm}^{-2} \mathrm{~s}^{-1}$

- Sensitivity beyond $1 \mathrm{TeV}$ already for 2011

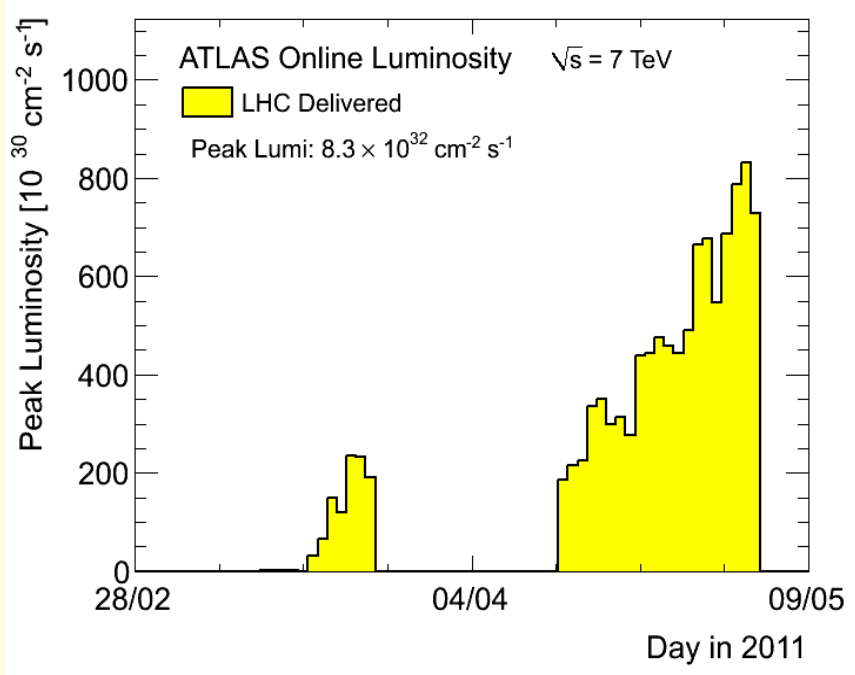

\section{This year is the SUSY year!}




\section{Many more results...}

https://twiki.cern.ch/twiki/bin/view/AtlasPublic/SupersymmetryPublicResults
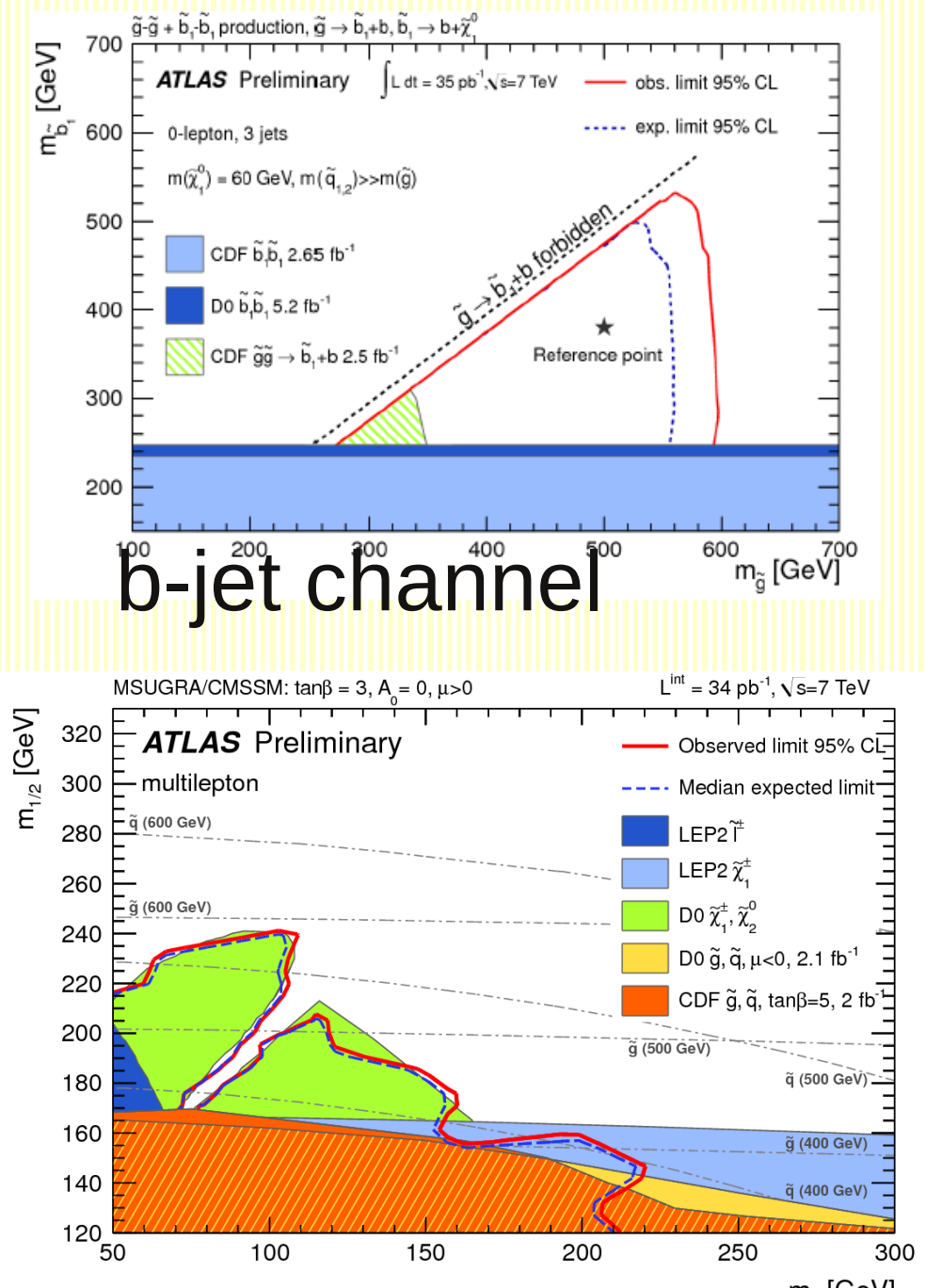

multilepton channel
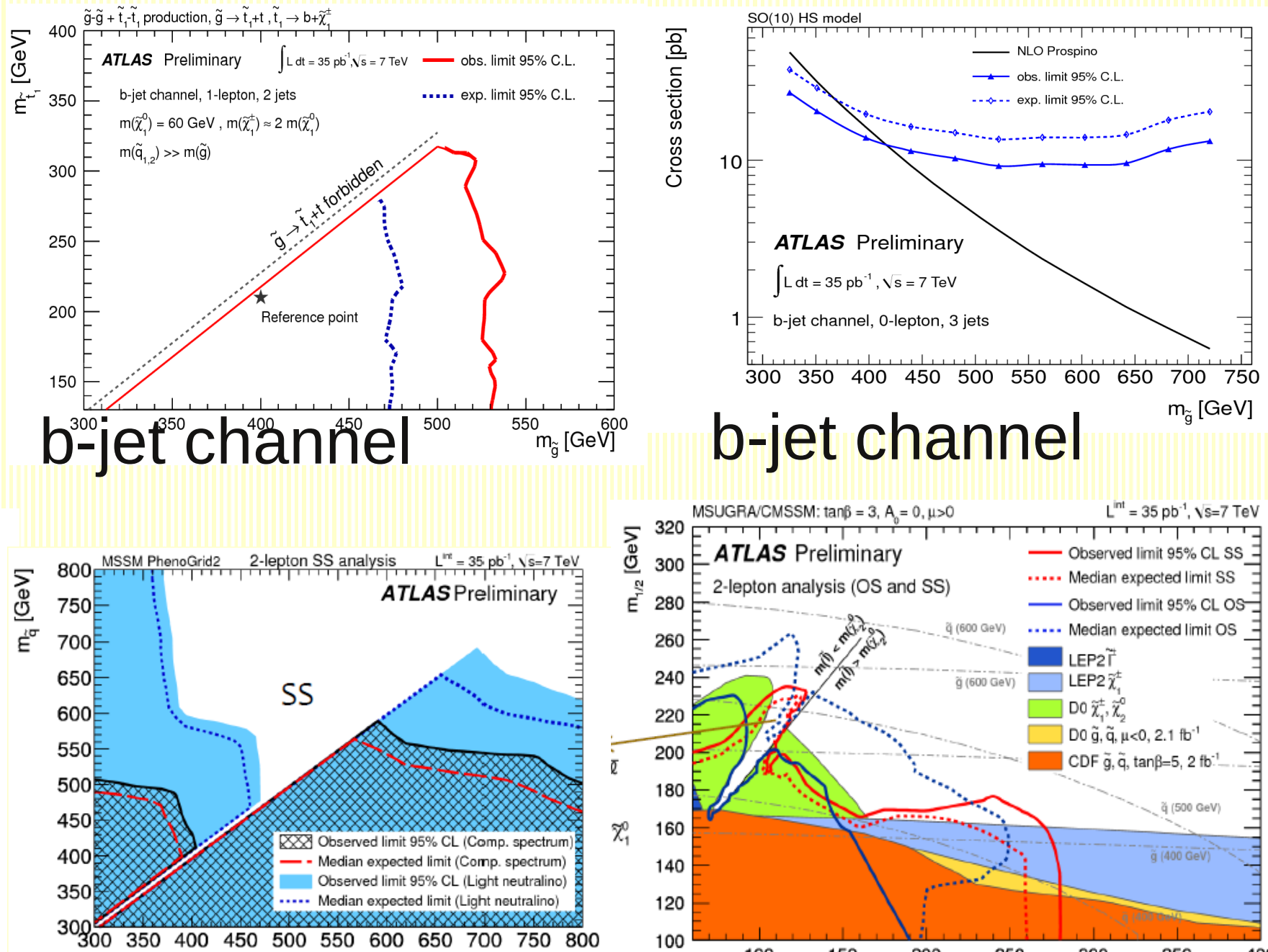

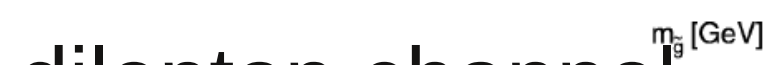

dilepton channel

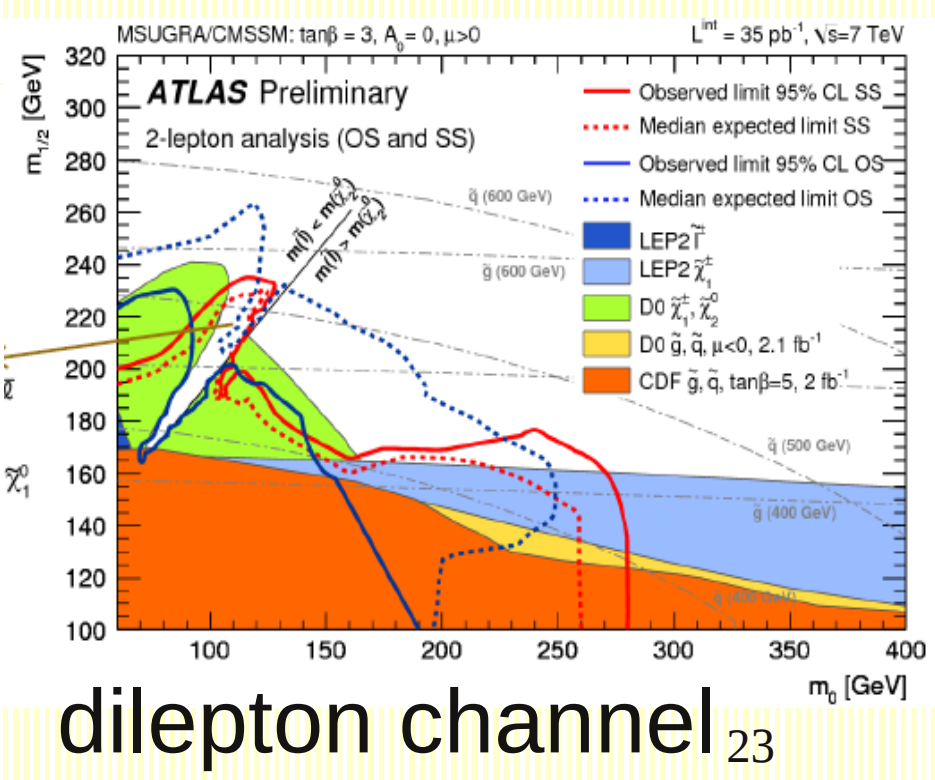




\section{Backup slides}




\section{Details of the results}

\begin{tabular}{lllll}
\hline \hline & Signal region A & Signal region B & Signal region C & Signal region D \\
\hline Total SM & $118 \pm 25[\mathrm{u}]_{-23}^{+32}[\mathrm{j}] \pm 12[\mathcal{L}]$ & $10.0 \pm 4.3[\mathrm{u}]_{-1.9}^{+4.0}[\mathrm{j}] \pm 1.0[\mathcal{L}]$ & $88 \pm 18[\mathrm{u}]_{-18}^{+26}[\mathrm{j}] \pm 9[\mathcal{L}]$ & $2.5 \pm 1.0[\mathrm{u}]_{-0.4}^{+1.0}[\mathrm{j}] \pm 0.2[\mathcal{L}]$ \\
Data & 87 & 11 & 66 & 2 \\
\hline
\end{tabular}

\begin{tabular}{lr}
\hline Electron channel & \multicolumn{1}{c}{ Signal region } \\
\hline Observed events & 1 \\
\hline Fitted top events & $1.34 \pm 0.52(1.29)$ \\
Fitted $W / Z$ events & $0.47 \pm 0.40(0.46)$ \\
Fitted QCD events & $0.0_{-0.0}^{+0.3}$ \\
\hline Fitted sum of background events & $1.81 \pm 0.75$ \\
\hline & Signal region \\
\hline Muon channel & 1 \\
\hline Observed events & $1.76 \pm 0.67(1.39)$ \\
\hline Fitted top events & $0.49 \pm 0.36(0.71)$ \\
Fitted $W / Z$ events & $0.0_{-0.0}^{+0.5}$ \\
Fitted QCD events & $2.25 \pm 0.94$ \\
\hline Fitted sum of background events
\end{tabular}




\section{SUSY production at LHC}
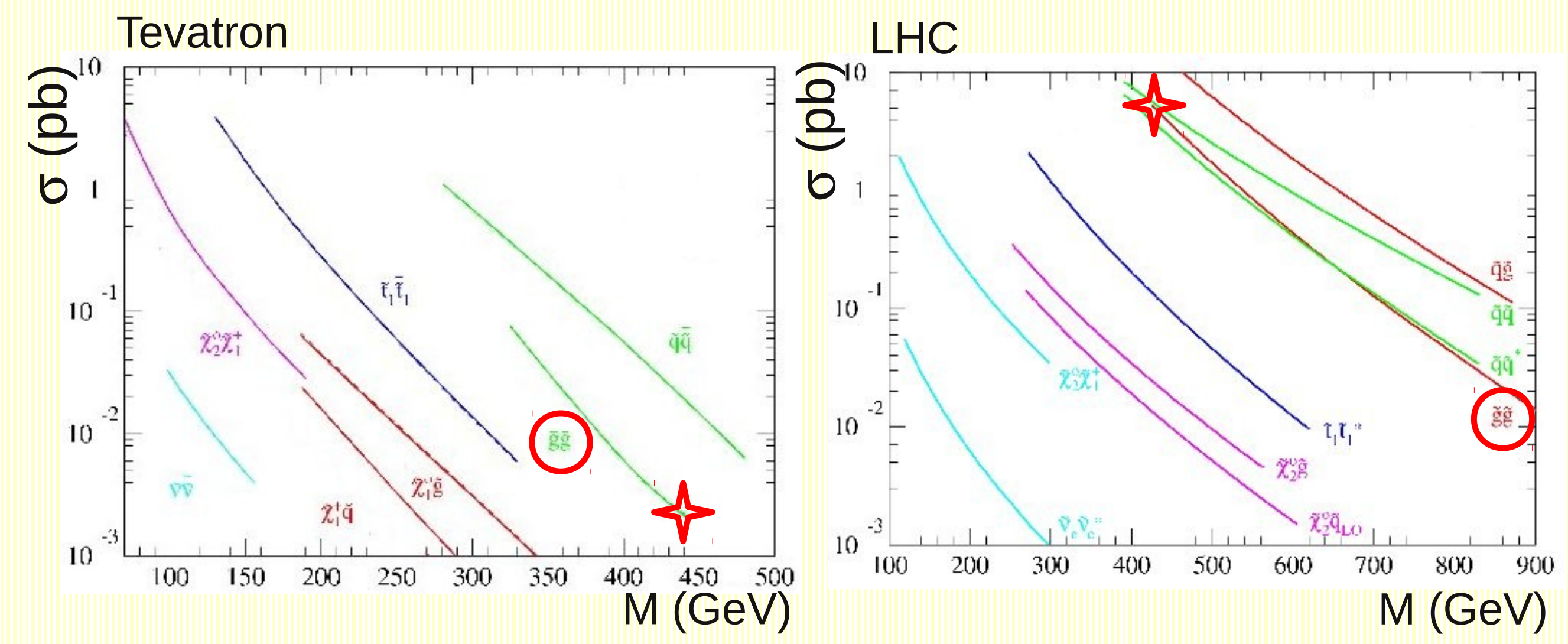


\section{SUSY production at LHC}

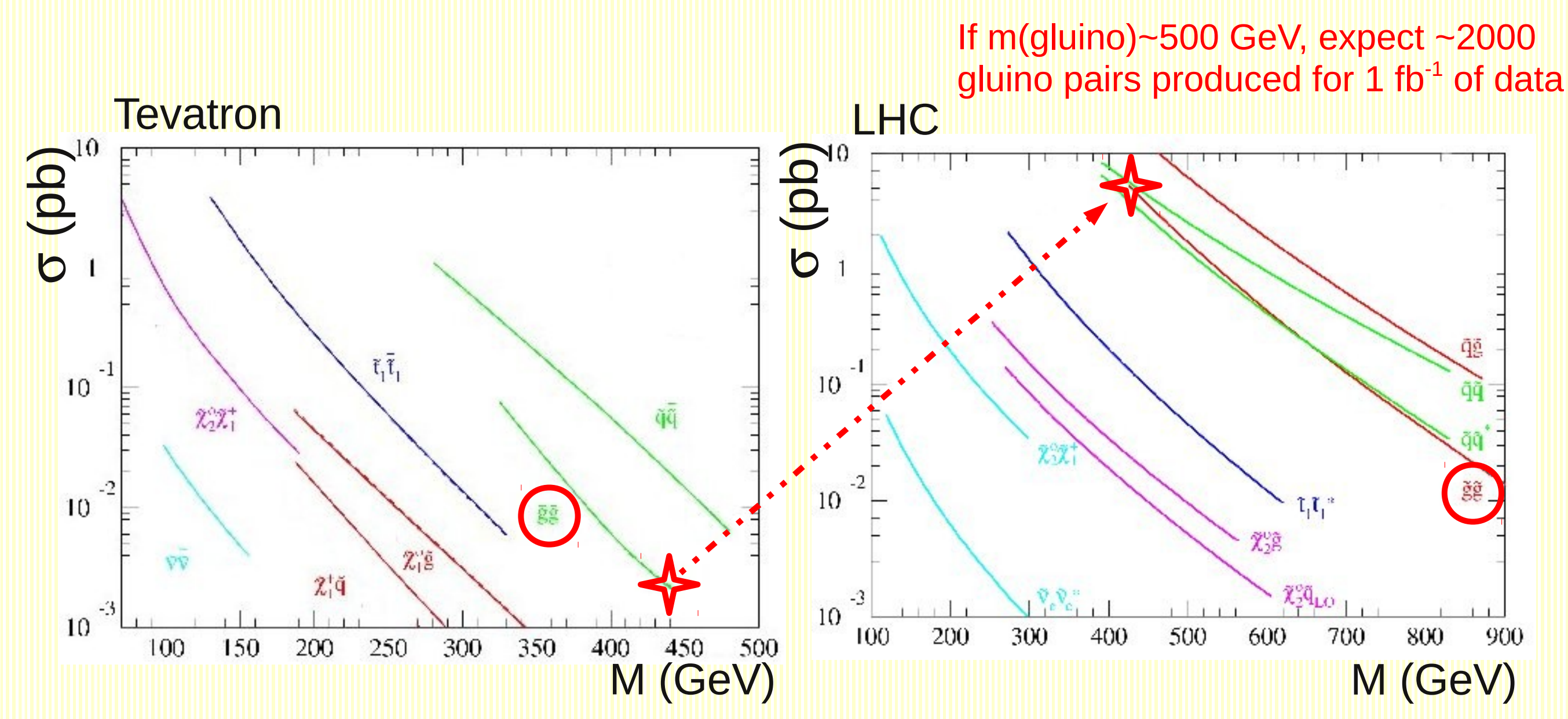

The cross sections for SUSY processes are way higher at LHC High-impact results possible with first data! 


\section{Object identification}

- Jets (anti-Kt, $R=0.4$ ): $p_{T}>20 \mathrm{GeV},|\eta|<2.5$

- Reject events compatible with noise or cosmics

- Remove if $\Delta \mathrm{R}$ (jet,electron) $<0.2$

$\Delta R=\sqrt{(\Delta \eta)^{2}+(\Delta \phi)^{2}}$

$\phi$ : azimuthal angle around the beam pipe $\eta=-\ln \tan (\theta / 2)$ where $\theta$ is the polar angle

- Electrons: $p_{T}>20 \mathrm{GeV},|\eta|<2.47$

- Outside problematic regions of the calorimeter

- Remove if $\Delta \mathrm{R}$ (jet,electron) $<0.4$

- Muons: $p_{\mathrm{T}}>20 \mathrm{GeV},|\eta|<2.4$, Sum $\mathrm{p}_{\mathrm{T}}$ of tracks $(\Delta \mathrm{R}<0.2)<1.8 \mathrm{GeV}$

- Remove if $\Delta \mathrm{R}$ (jet,muon) $<0.4$

- Missing transverse momentum $\left(E_{T}{ }^{\text {miss }}\right)$ :

- sum over the transverse momentum of all jets (up to $|\eta|<4.9$ ), electrons, muons and all calorimeter clusters not associated to such objects 


\section{$\mathrm{E}_{\mathrm{T}}{ }^{\text {miss }}$ performance}
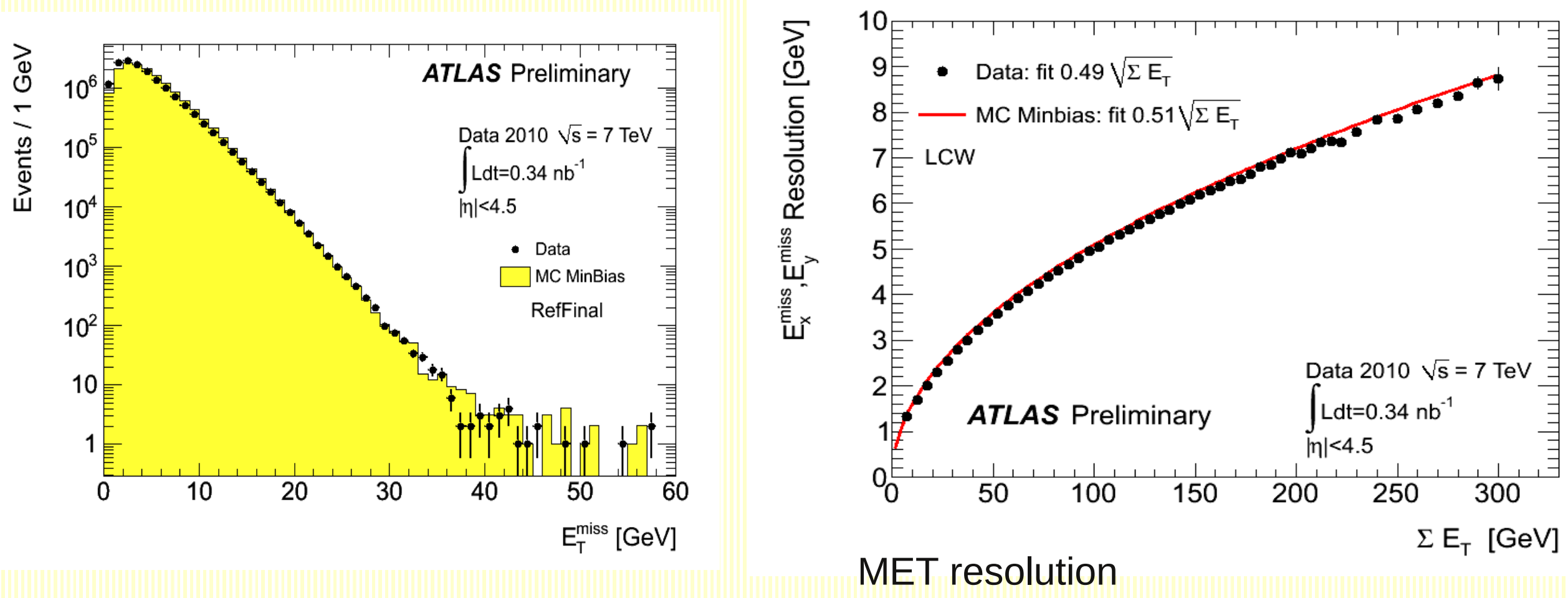


\section{QCD background: 1-lepton channel}

Evaluated using the 'matrix method' which plays on the difference in isolation between the leptons in QCD events with respect to signal leptons

- Loose control sample with isolation criteria relaxed with respect to the tight SUSY selections

- Define two categories: QCD leptons $(Q)$ and non-QCD leptons $(\not)$ $-\varepsilon$ is the probability that a loose lepton is also tight

$$
N_{\text {tight }}^{\text {obs }}=N_{\text {tight }}^{Q}+N_{\text {tight }}^{Q}
$$

$N_{\text {loose not tight }}^{\text {obs }}=\left(1 / \epsilon_{\not}-1\right) N_{\text {tight }}^{\not R}+\left(1 / \epsilon_{Q}-1\right) N_{\text {tight }}^{Q}$

The quantities in red are measured: solve the equations and extract the number of QCD events

electron channel

$N_{\text {tight }}^{Q}<0.3$ muon channel

$N_{\text {tight }}^{Q}<0.5$ 


\section{QCD background: 0-lepton channel}

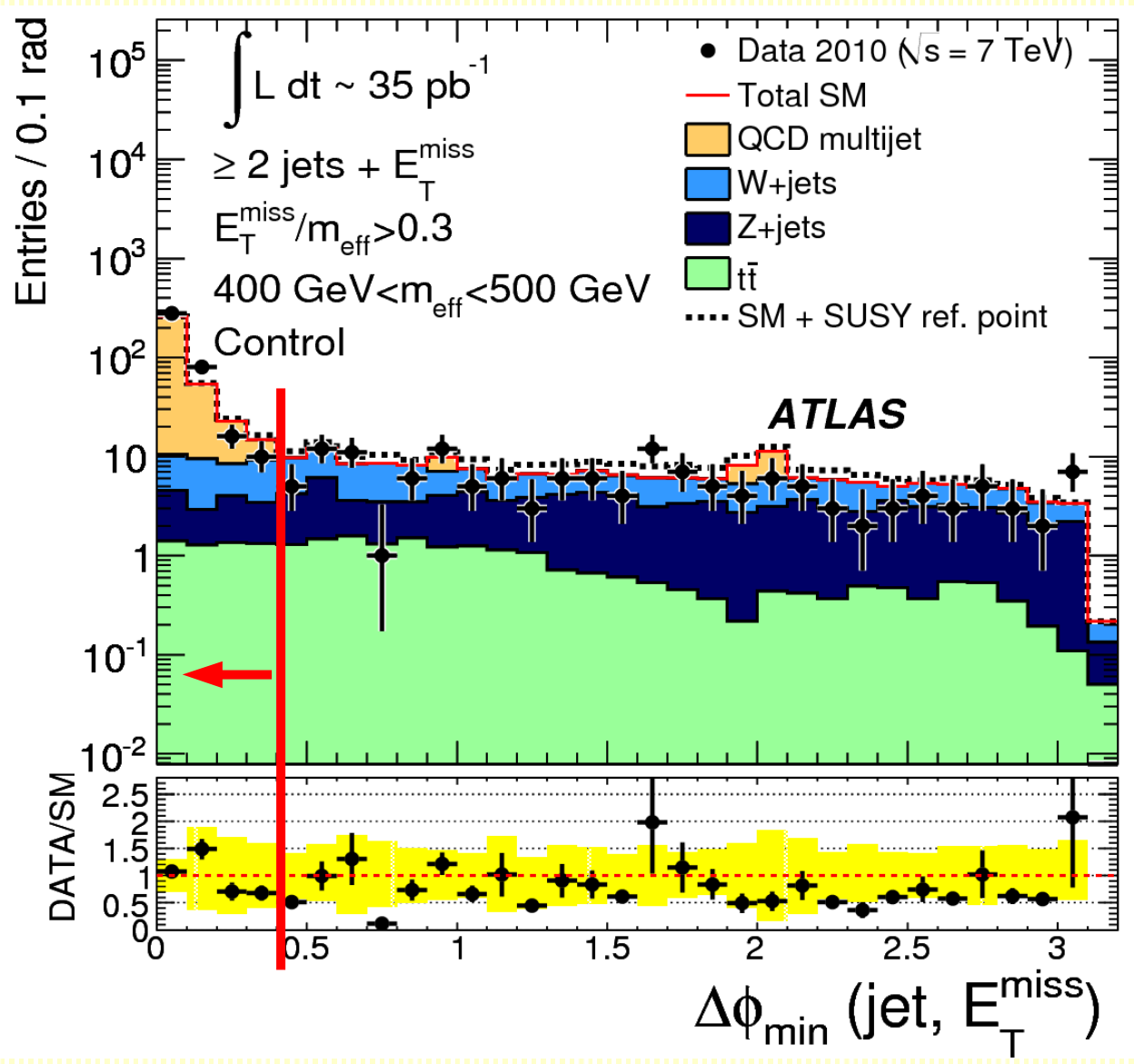

Partially data-driven estimate:

rescale the $\mathrm{MC}$ in the control region defined by reversing the $\Delta \phi$ cut

$<10 \%$ of the total BG

(cross checked with two other methods)
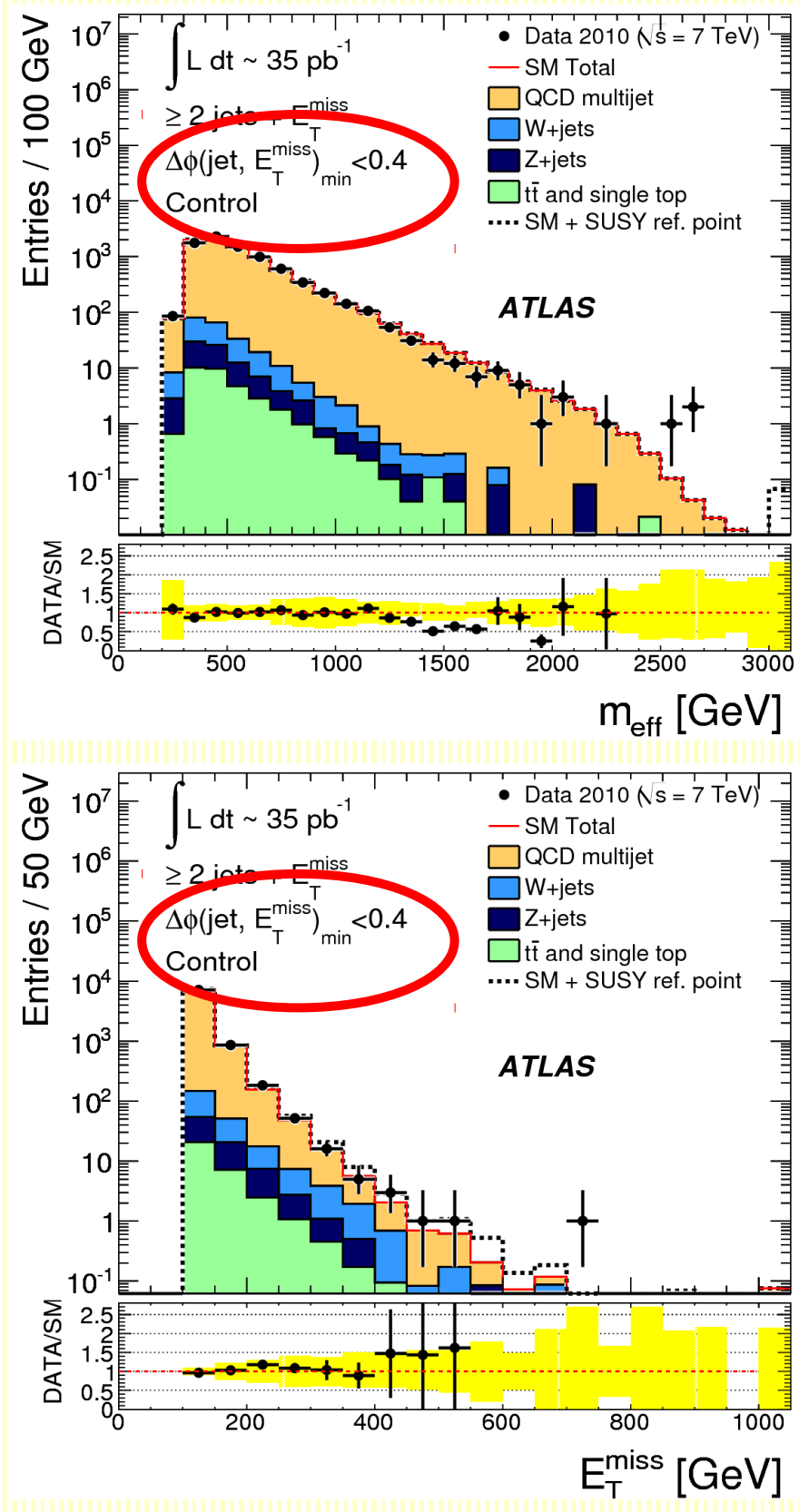


\section{Systematic uncertainties in the 1- lepton channel}

- Background:

- MC modeling of the $E_{t}^{\text {miss }}$ and $m_{T}$ distributions (variation of $M C$ generator and internal generator parameters)

- Finite statistics in the control regions

- Experimental uncertainties dominated by the jet energy scale, b-tagging and luminosity uncertainties

- Signal:

- Variation of the factorization and renormalization scales in Prospino

- PDF uncertainties (eigenvector sets provided by CTEQ6)

- Calculated separately for each production process 


\section{Limit setting}

$$
\begin{aligned}
L\left(\boldsymbol{n}, \boldsymbol{\theta}^{0} \mid \mu, \boldsymbol{b}, \boldsymbol{\theta}\right) & =P_{\mathrm{SR}} \times P_{\mathrm{CR}} \times P_{\text {Syst }} \\
& =P\left(n_{S} \mid \lambda_{S}(\mu, \boldsymbol{b}, \boldsymbol{\theta})\right) \times \prod_{i \in \mathrm{CS}} P\left(n_{i} \mid \lambda_{i}(\mu, \boldsymbol{b}, \boldsymbol{\theta})\right) \times P_{\text {Syst }}\left(\boldsymbol{\theta}^{0}, \boldsymbol{\theta}\right)
\end{aligned}
$$

The statistical treatment is based on the profile LLR, defined in the usual way as

$$
\Lambda(\mu) \equiv \Lambda\left(\mu, \boldsymbol{n}, \boldsymbol{\theta}^{0}\right) \equiv-2\left(\ln L\left(\boldsymbol{n}, \boldsymbol{\theta}^{0} \mid \mu, \hat{\hat{\boldsymbol{b}}}, \hat{\boldsymbol{\theta}}\right)-\ln L\left(\boldsymbol{n}, \boldsymbol{\theta}^{0} \mid \hat{\mu}, \hat{\boldsymbol{b}}, \hat{\boldsymbol{\theta}}\right)\right.
$$

where $\hat{\boldsymbol{\mu}}, \hat{\boldsymbol{b}}, \hat{\boldsymbol{\theta}}$ maximize the likelihood function, and $\hat{\boldsymbol{b}}, \hat{\boldsymbol{\theta}}$ maximize the likelihood for the specific, fixed value of the signal strength $\mu$, and the data $\boldsymbol{n}, \boldsymbol{\theta}^{0}$.

- The 1-sigma exclusion band has the same meaning as in a typical Higgs analysis, which show the upper limit on the $x$-section (but here the $x$-section is mapped on the $\mathrm{m} 0, \mathrm{~m} 12$ plane): any observation of number of events in the signal region that agrees with the bkg only prediction within $68 \% \mathrm{CL}$, gives an observed exclusion limit that lies within the 1-sigma uncertainty band.

- It can also be seen to correspond roughly to the $68 \%$ and $99 \%$ CL exclusion lines. The limit and bands have been obtained directly with toy MC. The coverage is therefore guaranteed to be exact. 